\title{
Mobile Learning in der Hochschullehre
}

\author{
Daniela Schmitz, Dominik May \& Karsten Lensing
}

\section{Einleitung}

Im täglichen Leben der Studierenden nehmen mobile Endgeräte eine zunehmend wichtigere Rolle ein. Die Anzahl derer, die weder ein Handy, noch einen MP3-Player oder einen Laptop besitzen, ist verschwindend gering ${ }^{(1)}$. Seit einigen Jahren steigt auch der Marktanteil von Smartphones und TabletPCs immer weiter an. 'Dabei ist die Nutzung dieser Endgeräte breit gefächert und reicht vom Abrufen des aktuellen Wetters, der Zugverbindungen oder der persönlichen E-Mails von unterwegs bis hin zum Ersatz eines Laptops durch einen Tablet-PC. Gegenüber dieser Entwicklung wird deutlich, dass die aktive Nutzung mobiler Endgeräte in der Lehre an Hochschulen noch in den Kinderschuhen steckt.

Erste wissenschaftliche Publikationen mit Definitionen zu Mobile Learning (kurz m-learning oder auch Wireless Learning, Ubiquitous Learning, Seamless Learning, Nomadic Learning oder auch Pervuasive Learning bzw. Educationii) lassen sich seit dem Jahr 2000 finden. Anhand der jeweils formulierten Definitionen lassen sich auch die Entwicklungsschritte der Fachdiskussion im Kontext des Mobile Learning nachvollziehen. Während Quinn (2000) davon spricht, dass es sich bei Mobile Learning um „elearning through mobile computational devices: Palms, Windows CE machines, even your digital cell phone" "III handelt, zeichnen Sharples et al. (2010) ein deutlich komplexeres Bild von Mobile Learning, indem sie schreiben, dass Mobile Learning auch durch die Mobilität von Lernenden und Wissen sowie durch die Kontextualisierung der Lernprozesse in die Lernenden-Umgebung charakterisiert wird.iv

Deutlich wird an dieser Gegenüberstellung auch, dass es möglich ist, sich dem Mobile Learning von unterschiedlichen Seiten zu nähern. Auf der einen Seite ist eine rein technische Betrachtungsweise möglich, welche die mobilen Endgeräte und ihre Eigenschaften in den Fokus nimmt und somit die technologische Dimension beschreibt. Auf der anderen Seite steht die didaktische Dimension, welche den Lernprozess und dessen Kontextualisierung sowie seine soziale Komponente im Fokus

(1) Um eine Datenbasis genau für diese Aussage zu erhalten, findet im Moment eine Umfrage zum Mediennutzungsverhalten im Rahmen des Forschungsprojekts „ELLI“ (www.elli-online.net) an den drei Hochschulen RWTH Aachen University, Ruhr-Universität Bochum und TU Dortmund statt. Mit Ergebnissen ist in der zweiten Jahreshälfte von 2013 zu rechnen. hat.v Eine erste, intern am Zentrum für HochschulBildung der TU Dortmund zu Mobile Learning durchgeführte Literaturanalyse betrachtet insgesamt 238 zumeist englischsprachige, wissenschaftliche Publikationen. Die fünf am häufigsten referenzierten Definitionen zu Mobile Learning stellen folgende Aspekte in den Vordergrund (Kursivierung von den Autoren dieses Beitrags ergänzt):

Mobile Learning devices are defined as handheld devices and [...] should be connected through wireless connections that ensure mobility and flexibility. ${ }^{\mathrm{vi}}$

... provides the potential of personal mobile technologies that could improve lifelong learning programs and continuing adult educational opportunities. $^{\text {vii }}$

What is new in >mobile learning comes from the possibilities opened up by portable, lightweight devices that are sometimes small enough to fit in a pocket or in the palm of the one's hand. viii

Any sort of learning that happens when the learner is not at a fixed, predetermined location, or learning that happens when the learner takes advantage of the learning opportunities offered by mobile technologies. ix

Learning will move more and more outside of the classroom and into the learner's environments, both real and virtual [...] new learning is highly situated, personal, collaborative and long term; in other words, truly learner-centered learning. [...] mobile devices are finding their way into classrooms in children's pockets, and we must ensure that educational practice can include these technologies in productive ways. ${ }^{x}$

Aufbauend auf diesen und weiteren Definitionen arbeitet Maske (2011) vier maßgebliche charakteristische Eigenschaften des Mobile Learning heraus, die sich in einem vierdimensionalen Beschreibungscluster für Mobile Learning Lehrveranstaltungen darstellen lassen (s. Abb. 1). ${ }^{\mathrm{xi}}$

Dieses soll im Folgenden zur Erläuterung der Beispiele herangezogen werden. Das heißt bei der Beschreibung von Mobile Learning Veranstaltungen werden die folgenden Fragen gestellt: ,Inwiefern ist die Nutzung mobiler Endgeräte in die Lehrveranstaltung eingeplant?“, „Inwiefern sind die Lernenden ortsunabhängig bei der Teilnahme an der Lehrveranstaltung?“, „Inwiefern sind die Lernprozesse 


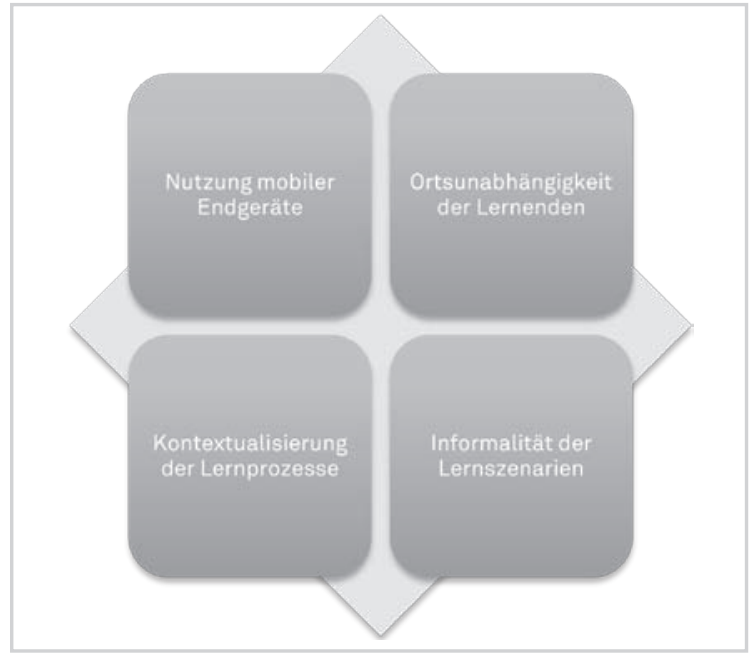

Abb. 1: Beschreibungscluster für Mobile Learning Lehrveranstaltungen

kontextualisiert?“(2)xii und „Inwiefern sind die Lernszenarien informell?"

\section{Praxisbeispiele Mobile Learning auf Exkursio- nen}

Die folgenden Praxisbeispiele entstanden auf der Basis der Zielvorgabe, Lernprozesse mit mobilen Endgeräten im Kontext der Hochschullehre anzureichern und bei der Realisierung keinen großen technischen Aufwand bzw. möglichst geringe Kosten zu verursachen. Im Folgenden stellen wir zwei schlanke (im Sinne von kostenneutralem und geringem technischen Aufwand), didaktische Einsatzszenarien dar, xii die als Ergänzung oder Begleitung zur Präsenzlehre einsetzbar sind. Dies sind nur zwei mögliche Lernszenarien aus einem breiten Spektrum, welches durch mobile Lerntechnologie ermöglicht wird.

Worauf kommt es also an, wenn Sie als Lehrende ihre Lehre mit Mobile Learning anreichern möchten? Zunächst sollte geprüft werden, ob ein sinnvoller Einsatz von Mobile Learning zur Erreichung der Lernziele (1) notwendig und (2) überhaupt möglich ist. Weiterhin ist zu klären, ob mobile Lernsequenzen zusätzlich unterstützende oder notwendigerweise zu absolvierende Lernsequenzen sind und welche Mischung von Präsenz-, Online- und Mobile-Lernphasen vorgesehen sind. Als förderliche Faktoren für mobile Lernszenarien bieten sich

(2) Nach Göth, Frohberg und Schwabe lässt sich Mobile Learning in vier Kontexte einteilen: 1) in irrelevante Kontexte wie in Bus oder Bahn, wo der Kontext für das Lernen keine besondere Rolle spielt; 2) Lernen in formalisierten Kontexten wie in der Vorlesung oder im Seminar, wo das Lernen in einen institutionalisierten Kontext mit formalen Abläufen eingebunden ist; 3 ) in sozialen Kontexten, wo das Lernen in Lerngruppen von Bedeutung ist; 4) in physischen Kontexten, wo Lernkontext und Umgebung stimmig sind wie beispielsweise das Lernen im Museum (vgl. Göth et al. 2007, S. 13). offene Aufgabenstellungen an, die unter anderem ein Lernen in kleineren Gruppen ermöglichen und auch Raum für informelle Lernprozesse lassen. Bei der Gestaltung mobiler Lernszenarien sollte nicht vergessen werden, dass durch die Nutzung der persönlichen Geräte der Studierenden das Lernen in den privaten Kontext übergeht. Die Nutzung eines eigenen Gerätes bringt eine höhere affektive Bindung mit sich und sollte daher nicht unreflektiert erfolgen. Zudem sollte bei der Gestaltung vor allem der mobile Ansatz der Lerntechnologie ausgereizt werden, um ein mobiles Endgerät nicht für eLearning einzusetzen, welches genauso gut mit Hilfe eines PCs durchzuführen wäre. Letztendlich sollte die Zielgruppe beachtet werden, da nicht alle Studierenden standardmäßig mit dem mobilen Endgerät immer und überall für jede Lehrveranstaltung lernen (wollen).

\subsection{Dokumentation und Reflexion des Lernprozes-} ses

Mit Hilfe von Textverarbeitungsapplikationen für mobile Endgeräte, die kostenfrei erhältlich und plattformunabhängig sind, halten die Studierenden zu Beginn einer Veranstaltung oder einer Lerneinheit ihre persönlichen Lernziele fest. Dies können neben inhaltlichen auch soziale und methodische Ziele sein. Während einer Exkursion machen sich die Studierenden beispielsweise Notizen zu den Inhalten und ihren Lernzielen und dokumentieren, inwieweit sie ihre persönlichen Lernziele erreicht haben. Zum Abschluss der Lerneinheit werden diese Ziele thematisiert und der Lernprozess wird reflektiert. Mobile Endgeräte fungieren hier als individuelle, mobile Lernprozessunterstützung, zum Beispiel bei Museumsbesuchen oder Betriebsbesichtigungen im Rahmen kleinerer Seminare oder Projektgruppen. Neben Vorgaben zum Lerninhalt können die Studierenden durch die Internetanbindung auch erweiterte Informationen abrufen und adhoc entstehende Lernbedarfe stillen. Die Realisierung ist mit einer simplen Dokumentenvorlage im txt-Format möglich, welche Vorstrukturierungen für die Notizen oder Satzanfänge für die Lernziele enthält. Diese Vorlage erhalten die Studierenden vom Lehrenden und können diese auf ihre Geräte laden.

Beispielszenario: In der Vorbesprechung am Morgen des Exkursionstages legen die Lernenden ihre Lernziele für den Tag fest. Während des Tages gibt es vor den Pausen die Aufforderung zu dokumentieren und zu prüfen, inwieweit die Lernziele schon erreicht wurden und wie der Lernweg verlaufen ist. In der Nachbesprechung werden die Lernzielerreichung und der individuelle Lernweg reflektiert.

Einordnung in das Mobile Learning Cluster: Der Fokus dieses Beispielszenarios liegt in der Kontex- 
tualisierung der individuellen Lernprozesse. Das Lernen findet direkt im relevanten physischen Kontext statt. Durch die Lernform der Exkursion besteht nur eine relative Ortsunabhängigkeit zwischen einzelnen Lernstationen. Das Lernen kann aber nicht außerhalb des Exkursionsortes stattfinden, da der relevante Kontext vor Ort eben das Lernen ermöglicht. Anders sieht die begleitende Dokumentation und Reflexion des Lernprozesses im Rahmen einer klassischen Präsenzlehrveranstaltung aus. Dabei kann das mobile Lernen als dokumentierte Reflexion des Lernens ortsunabhängig und in einem beliebigen Kontext stattfinden. Informelles Lernen didaktisch in einem Lernszenario direkt gestalten zu wollen, erweist sich als schwierig. Informelles Lernen ist interessengesteuert und muss nicht mit den Inhalten der Lehrveranstaltung identisch sein. Sicherlich sind im Rahmen eines Museumsbesuches neben dem Lehrziel der Veranstaltung zahlreiche Möglichkeiten gegeben, informell zu lernen.

\subsection{Generierung von Lernfragen}

Dieser Ansatz bringt im Unterschied zum ersten einen Perspektivwechsel für die Lernenden mit sich. Die Lernenden werden in einem Lernsetting zu einem Perspektivwechsel dazu angeregt, vom Lernenden in die Rolle des Lehrenden zu schlüpfen. $\mathrm{Zu}$ vorab definierten Lerneinheiten sollen die Lernenden Lernfragen bzw. Übungsaufgaben mit den richtigen Antworten bzw. Lösungshinweisen generieren (je nach Möglichkeit kann auch schon eine Auswahl an falschen Antworten dazu generiert werden). Diese Lernfragen kommen dann zur inhaltlichen Nachbereitung von Exkursionen oder einer Seminareinheit zum Einsatz. Die Gruppen tauschen ihre Lernfragen aus, erhalten gegenseitig Einblicke in die Inhalte und Interessensschwerpunkte der anderen Gruppen und bereiten so den Lernstoff der Lerneinheit nach.

Beispielszenario: Die Lernenden fertigen zu einem Ausstellungsbereich des Museums Lernfragen an. Inhalte aus diesem Bereich, die die Studierenden für interessant und lehrreich befinden, werden in Frageform verfasst. Neben der richtigen Antwort müssen beispielsweise auch drei falsche Antworten gefunden werden. Diese von den Lernenden erstellten Lernfragen dienen der Nachbereitung der Exkursion vor Ort, indem die einzelnen Lerngruppen ihre Lernfragen austauschen.

Einordnung in das Mobile Learning Cluster: Dieses Lernszenario nutzt mobile Lerntechnologie begleitend und ortsunabhängig für die Erstellung der Lernfragen. Der Kontext des Lernens kann entweder irrelevant sein oder in der Gruppe in einem sozialen Kontext stattfinden. Findet das Lernszenario als Exkursion statt, wäre es ein für das Lernen relevanter physischer Kontext. Aus diesem Lernszenario kann informelles Lernen entstehen, wenn beispielsweise Lernende ihre Lernfragen interessierten Personen auch außerhalb der Exkursionsgruppe über ein Forum o.ä. zur Verfügung stellen.

\section{Teaching Tips und Ausblick}

Wenn Sie mobile Lernszenarien umsetzen möchten, sollten Sie auch potentielle Probleme berücksichtigen. Denn neben technischen und didaktischen Aspekten können zusätzlich weitere unvorhergesehene Aspekte als potentielle Störfaktoren für das Lernszenario auftauchen:

- Der Faktor „Draußen“: Wettereinflüsse, Verfügbarkeit des Lernangebotes

- Der Faktor Technik: Internetzugang, Akkulaufzeit, Funktion der Geräte und Anwendungen, Nutzung bestehenden Contents oder Contenterstellung, Anbindung an bestehende Technologien wie Lernplattformen

- Der Faktor Lernende: Motivation, Lernbereitschaft mobil zu lernen, Einstellung zu mobilen Endgeräten

- Der Faktor Lehrende: Kompetenzen zur Contenterstellung und -aufbereitung

Resümierend lassen sich aus den bisherigen Überlegungen und vorgestellten Beispielen so genannte Do's and Dont's für Lehrende festhalten. Die folgende Tabelle fasst die Tipps stichpunktartig zusammen:

\begin{tabular}{|l|l|}
\hline Do's & Dont's \\
\hline $\begin{array}{l}\text { Informelles Lernen in Klein- } \\
\text { gruppen ermöglichen }\end{array}$ & $\begin{array}{l}\text { Zu starr vorgegebene Auf- } \\
\text { gaben }\end{array}$ \\
\hline $\begin{array}{l}\text { Selbstorganisierte Heran- } \\
\text { gehensweisen bei der Bear- } \\
\text { beitung }\end{array}$ & $\begin{array}{l}\text { Ausschließlich mit Leihgerä- } \\
\text { ten für die gesamte Lerngrup- } \\
\text { pe arbeiten }\end{array}$ \\
\hline $\begin{array}{l}\text { Sinnvolle Einbindung ins Ge- } \\
\text { samtkonzept der Lehrveran- } \\
\text { staltung }\end{array}$ & $\begin{array}{l}\text { Auf Reflexion des Lernwegs } \\
\text { und des Technologieeinsatzes } \\
\text { verzichten }\end{array}$ \\
\hline $\begin{array}{l}\text { Notwendigkeit der mobilen } \\
\text { Lernunterstützung prüfen }\end{array}$ & $\begin{array}{l}\text { Nur als eLearning auf dem Ta- } \\
\text { blet umsetzen }\end{array}$ \\
\hline $\begin{array}{l}\text { Abwechslungsreicher } \\
\text { Medieneinsatz, verschiedene } \\
\text { Lerntypen ansprechen }\end{array}$ & $\begin{array}{l}\text { Voraussetzen, dass alle Stu- } \\
\text { dierende „Digital Natives“ } \\
\text { sind }\end{array}$ \\
\hline
\end{tabular}

Das Thema „Mobile Learning“ wird am Zentrum für HochschulBildung im Rahmen des Forschungsprojekts „ELLI - Exzellentes Lehren und Lernen in den Ingenieurwissenschaften“ fokussiert. Neben der Entwicklung und Erprobung von Mobile Learning Anwendungsszenarien geht es auch um deren Einbettung in den Gesamtkontext von virtueller Lehre. Für die hochschuldidaktische Forschung zur Nutzung mobiler Endgeräte in der Lehre sind zurzeit vier konkrete Szenarien in Planung bzw. bereits in der Umsetzung. (1) Neben den mobilen Endgeräten werden im Rahmen von ELLI und in Zusammenarbeit mit dem „Institut für Umformtechnik und Leichtbau“ (IUL) auch Lehr-Lernszenarien mit Remote Laboratories (am IUL sind dies im Speziellen Labore 
mit entsprechendem Equipment zur Bestimmung und Untersuchung von Materialkennwerten, welche über einen online-Zugang nutzbar sind) erarbeitet. Mobile Learning soll in diesen Kontext integriert werden. Das heißt, dass die Labore zukünftig auch über mobile Endgeräte nutzbar gemacht werden. In Ergänzung dazu wird auch an einem Mobile-Learning-Szenario gearbeitet, welches die Reflexion des Lernens und die Kollaboration der Studierenden mit Hilfe der mobilen Endgeräte fördert. ${ }^{\text {xiv }}$ (2) Ein weiteres Szenario wird im Kontext einer transnationalen online-Lehrveranstaltung zwischen der TU Dortmund und der University of Virginia umgesetzt. Im Rahmen dieser Lehrveranstaltung lernen deutsche und amerikanische Studierende gemeinsam, indem sie die gleiche Lehrveranstaltung online besuchen und dort gemeinsame Lehrprojekte durchführen. ${ }^{x v}$ In Zukunft soll erforscht werden, welche Vorteile für die Kommunikation und Kollaboration die Nutzung mobiler Endgeräte birgt und welchen Einfluss dies auf die Gestaltung des Lehr-Lernszenarios in der praktischen Umsetzung hat. (3) Für eine Veranstaltung zum Thema „Projektmanagement“ wird zurzeit untersucht, wie effektive Kommunikation im Projekt durch die Nutzung mobiler Endgeräte unterstützt werden kann. Für diese Untersuchung wurden Studierende mit entsprechender Hardware ausgestattet und diese erarbeiten in Kooperation mit den Dozierenden ein Lastenheft, welches die notwendigen Ausstattungsmerkmale eines mobilen Endgeräts beschreibt, um Projektkommunikation zu fördern. (4) Ein letztes Szenario zielt auf die Kreativität von Studierenden ab und wird in einer Veranstaltung zu Kreativität selbst umgesetzt. Mit Hilfe einer speziell entwickelten App sollen Studierende ihre persönlichen, kreativen Momente im alltäglichen Kontext mit Hilfe von Fotos, kurzen Sprachnotizen o.ä. festhalten. Ausgehend davon wird einerseits untersucht, wann Studierende kreativ sind, anderseits sollen die Studierenden durch das Teilen und Reflektieren dieser Momente in einen gemeinsamen, kreativen Prozess kommen.

Die oben beschriebenen Szenarien sind nur Beispiele für eine Unzahl von Möglichkeiten, mobile Endgeräte in der Lehre einzusetzen. Die Forschung dazu hat gerade erst begonnen. Sollten Sie auch eine Idee haben und suchen dafür Unterstützung oder möchten diese intensiv beforschen, so freuen wir uns, wenn Sie sich mit uns in Kontakt setzen. Ansonsten können wir Sie nur ermuntern, Ihre Ideen in diesem Kontext einfach umzusetzen.

\section{Anmerkungen}

i Internetquelle: http://www.crn.de/hardware/artikel-98610.html (abgerufen am 21.05.2013)

ii Frohberg, D. (2008): Mobile-Learning. Dissertation, S. 3

iii Quinn, C. (2000): mLearning: Mobile, Wireless, in your Pocket Learning. LineZine, Fall 2000, URL: http://www. linezine.com/2.1/features/cqmmwiyp.htm Webdokument, letzter Zugriff: 21.05.2013

iv Sharples, M. et al (2010): A Theory of Learning for the Mobile Age. In Bachmair, B. (Hrsg.): Medienbildung in neuen Kulturräumen. Wiebaden: VS Verlag für Sozialwissenschaften, S. 87-99 zitiert nach Ph. Maske (2011): Mobile Applikationen 1 Interdisziplinäre Entwicklung am Beispiel des Mobile Learning. Wiesbaden: Springer Gabler, S. 152.

$\checkmark$ Maske, Ph. (2011): Mobile Applikationen 1 Interdisziplinäre Entwicklung am Beispiel des Mobile Learning. Wiesbaden: Springer Gabler, S. 154.

vi QUINN, C. (2001): mLearning: mobile, wireless, in-yourpocket learning, LineZine.

vii Sharples, Mike (2000): The Design of Personal Mobile technologies for Lifelong Learning, 34. Aufl.

viii Kukulska-Hulme, Agnes / Traxler, John (2005): Mobile learning. A handbook for educators and trainers. London.

ix O'Malley, Claire u.a. (2003): WP4-Guidelines for learning/ teaching/tutoring in a mobile environment/MO-Bllearn. Forschungsbericht.

${ }^{x}$ Naismith, Laura (2004): Literature Review in Mobile Technologies and Learning. (NESTA Futurelab Series, Report 11). In: NESTA Futurelab Series.

xi Maske, Ph. (2011): Mobile Applikationen 1 Interdisziplinäre Entwicklung am Beispiel des Mobile Learning. Wiesbaden: Springer Gabler, S. 152.

xii Göth, C. / Frohberg, D. / Schwabe, G. (2007): Von passivem zu aktivem mobilen Lernen. In: Zeitschrift für e-learning, Lernkultur und Bildungstechnologie, 2. Jg., H. 4, S. $12-28$.

xiii Schmitz, D. (2012): Mobile Lernprozessunterstützung auf Exkursionen. In: Hamburger eLMagazin \#09, Dezember 2012, S. 23-25, URL: http://www.uni-hamburg.de/ eLearning/eCommunity/Hamburger_eLearning_Magazin/eLearningMagazin_09.pdf

xiv Terkowsky, C. / May, D. / Haertel, T. / Pleul, C. (2013): Experiential Learning with Remote Labs and E-Portfolios - Integrating tele-operated experiments into personal learning environments. In: International Journal of Online Engineering (iJOE), Vol. 9, No. 1/2013, S. 12-20.

${ }^{x v}$ Moore, St. / May, D. / Wold, K. (2012): Developing Cultural Competency in Engineering through Transnational Distance Learning. In Hogan, R. (ed.): Transnational Distance Learning and Building New Markets for Universities. Hershey (PA/USA): IGI Global, S. 210-228.

\section{Autor/Autorin}

Daniela Schmitz, wissenschaftliche Mitarbeiterin im Projekt TUMENDO des Zentrums für HochschulBildung (zhb) der TU Dortmund.

E-Mail: daniela.schmitz@tu-dortmund.de

Dominik May und Karsten Lensing, Geschäftsführer bzw. studentischer Mitarbeiter im Kompetenz- und Dienstleistungszentrum für das Lehren und Lernen in den Ingenieurwissenschaften (TeachING-LearnING.EU).

E-Mail:may@teaching-learning.eu 